Short Communication

Bangladesh Agron. J. 2014, 17(2): 95-97

\title{
EFFECT OF NPK AND PLANT EXTRACTED PYROLIGNEOUS ACID ON YIELD OF Boro RICE
}

\author{
S. M. Masum ${ }^{1}$, M. H. Ali ${ }^{2}$, I. F. Chowdhury ${ }^{3}$, M. S. H. Mandal ${ }^{4}$ and M. N. Haque ${ }^{4}$ \\ ${ }^{1}$ Assistant Professor, ${ }^{2}$ Professor, ${ }^{3}$ Lecturer, ${ }^{4}$ Research Assistant, \\ Department of Agronomy, Sher-e-Bangla Agricultural University, Dhaka-1207, Bangladesh. \\ Corresponding author: smmasum607@yahoo.com
}

\begin{abstract}
Rice (Oryza sativa L.) is the staple food of Bangladesh. The yield of rice is low in Bangladesh than the other rice growing countries like China, Japan, South Korea and Vietnam where the average yield is $6.74,6.74,6.37$ and $5.66 \mathrm{t} \mathrm{ha}^{-1}$, respectively (IRRI, 2012). Deep placement of slow releasing nitrogenous fertilizer such as, urea super granule (USG) reduces loss as well as increases its use efficiency (Mohanty et al., 1989). USG requires only one-time application in rice or wheat cultivation, whereas surface application of $\mathrm{PU}$ requires two to three split applications resulted in significant $\mathrm{N}$ loss.
\end{abstract}

Wood vinegar or pyroligneous acid is a condensed liquid generated from the gas and combustion of fresh wood burning in airless condition by charcoal production. Wood vinegar can be applied to the soil surface to help increase the population of beneficial microbes and to promote plant root growth. PRH (a trade name) is a liquid solution which is extracted from fruits and other natural ingredients as like as vinegar or pyroligneous acid. It is $100 \%$ organic, can be used in rice, wheat, maize fields as well as in other cereal crops. It also increases the number of microorganisms present in soil and thus increase root growth through microbial activity. Masum et al. (2013) found that rice grain yield significantly increased when PRH was incorporated with $50 \%$ BRRI recommended dose of fertilizer for rice. Therefore, the present study was undertaken to to evaluate the efficacy of PRH in relation to urea super granule and recommended dose of fertilizers considering boro rice as a test crop.

A field experiment was conducted at the Agronomy research field of Sher-e-Bangla Agricultural University, Dhaka-1207 during the period from November, 2012 to May, 2013. The variety used in this experiment was BRRI dhan29. The experiment was laid out in a Randomized Complete Block Design ( $\mathrm{RCBD}$ ) with three replications. There are twelve treatments viz., $\mathrm{T}_{1}=$ Control (no NPK fertilizer), $T_{2}=$ No nitrogen fertilizer, $T_{3}=$ No phosphorus fertilizer, $T_{4}=$ No potassium fertilizer, $\mathrm{T}_{5}=50 \%$ recommended dose of granular urea and $50 \% \mathrm{PRH}$ (fruit vinegar), $\mathrm{T}_{6}=50 \%$ recommended dose of phosphorus fertilizer and 50\% PRH (fruit vinegar), $\mathrm{T}_{7}=50 \%$ recommended dose of potassium fertilizer and 50\% $\mathrm{PRH}$ (fruit vinegar), $\mathrm{T}_{8}=50 \%$ recommended dose of NPK fertilizer and 50\% PRH (fruit vinegar, prepared by $100 \mathrm{ml} \mathrm{PRH} \mathrm{in} 10$ litre of water), $\mathrm{T}_{9}=100 \%$ recommended dose of NPK fertilizer, $\mathrm{T}_{10}=100 \% \mathrm{PRH}$ (fruit vinegar, prepared by $200 \mathrm{ml} \mathrm{PRH}$ in $200 \mathrm{ml}$ of water), $\mathrm{T}_{11}=2.7 \mathrm{~g}$ urea super granules per 4 hills, $\mathrm{T}_{12}=$ $2.7 \mathrm{~g}$ urea super granules per 4 hills and 50\% PRH (fruit vinegar). Recommended dose of fertilizer for boro rice as Urea, TSP, MoP, Gypsum and Zinc Sulphate @ 120, 90, 40, 60 and $10 \mathrm{~kg} \mathrm{ha}^{-1}$, respectively. Urea super granules were applied @ $46 \mathrm{~kg} \mathrm{~N} \mathrm{ha}^{-1}$. All fertilizers were applied during final land preparation except urea which was applied in three equal splits at 7, 30 and 50 DAT. Foliar application of PRH also done in the same dates as urea applied. Urea super granules were placed at the centre of four hills of two adjacent rows at 10 DAT. Thirty day old seedlings were uprooted and transplanted in the field on December 10, 2012 and harvesting was done on May 10, 2013 manually from each plot. All the collected data were analyzed and the 
Masum et al.

mean differences among the treatments were compared by Duncan's Multiple Range Test (Gomez and Gomez, 1984).

Significant variation was observed in terms of effective tillers hill ${ }^{-1}$, non-effective tillers hill $^{-1}$, panicle length, filled grain, unfilled grain and 1000-grain weight (Table 1). It was observed that the highest (18.35) number of effective tillers hill ${ }^{-1}$ was observed from the treatment $T_{12}$ while other showed similar no. of tiller /plant but was statistically similar with other treatments except $\mathrm{T}_{12}$. The maximum panicle length was produced from $\mathrm{T}_{7}$ and $\mathrm{T}_{9}$ produced the lowest $(20.13 \mathrm{~cm})$ . The highest (111.70) number of filled grains panicle/ panicle was recorded from $\mathrm{T}_{12}$ while control $\left(\mathrm{T}_{1}\right)$ produced the highest (12.94) number of unfilled grains panicle / panicle. There was no definite trend observed in 1000- grain weight. The highest grain yield $\left(6.61 \mathrm{t} \mathrm{ha}^{-1}\right)$ was observed with urea super granules $(2.7 \mathrm{~g}$ ) per 4 hills and 50\% PRH (fruit vinegar) by $98.51 \%$ than the control $\left(T_{1}\right)$ and by $35.73 \%$ from the $100 \%$ recommended dose of NPK fertilizers $\left(T_{9}\right)$. Application of $50 \%$ recommended dose of NPK fertilizer and 50\% PRH (fruit vinegar) $\left(\mathrm{T}_{8}\right)$ increased grain yield of rice by $40.30 \%$ than control $\left(\mathrm{T}_{1}\right)$. Kabir et al. (2009), Masum et al. (2008) and BRRI (2000) reported that application of USG produces higher rice grain yield than prilled urea. The highest straw yield $\left(7.90 \mathrm{t} \mathrm{ha}^{-1}\right)$ was produced by the treatment $\mathrm{T}_{12}$ which was statistically similar with $T_{1}$ while other treatments were similar in straw yield.

Table 1. Effect of different fertilizers rate and PRH on yield components of boro rice var. BRRI dhan29

\begin{tabular}{|c|c|c|c|c|c|c|c|c|}
\hline Treatments & $\begin{array}{c}\text { Effective } \\
\text { tillers } \\
\text { hill }^{-1} \\
\text { (no.) }\end{array}$ & $\begin{array}{c}\text { Non } \\
\text { effective } \\
\text { tillers hill }{ }^{-1} \\
\text { (no.) }\end{array}$ & $\begin{array}{l}\text { Panicle } \\
\text { length } \\
\text { (cm) }\end{array}$ & $\begin{array}{c}\text { Filled grains } \\
\text { panicle }^{-1}\end{array}$ & $\begin{array}{c}\text { Unfilled } \\
\text { grains } \\
\text { panicle }{ }^{-1}\end{array}$ & $\begin{array}{c}1000- \\
\text { grain } \\
\text { weight } \\
\text { (g) }\end{array}$ & $\begin{array}{c}\text { Grain } \\
\text { yield } \\
\left.\text { (tha }^{-1}\right)\end{array}$ & $\begin{array}{c}\text { Straw } \\
\text { yield } \\
\left(\text { tha }^{-1}\right)\end{array}$ \\
\hline $\mathrm{T}_{1}$ (Control) & $13.73 \mathrm{~b}$ & $5.60 \mathrm{a}$ & $20.77 \mathrm{ab}$ & $105.60 \mathrm{~d}$ & $12.94 \mathrm{a}$ & $22.40 \mathrm{~cd}$ & $3.35 e$ & 7.27ab \\
\hline $\mathrm{T}_{2}$ & $13.33 \mathrm{~b}$ & $4.80 \mathrm{ab}$ & $20.91 \mathrm{ab}$ & $107.60 \mathrm{~b}-\mathrm{d}$ & $10.54 \mathrm{~b}-\mathrm{d}$ & $22.87 \mathrm{~b}-\mathrm{d}$ & $3.80 e$ & $6.88 \mathrm{~b}$ \\
\hline $\mathrm{T}_{3}$ & $14.23 \mathrm{~b}$ & $5.60 \mathrm{a}$ & $22.07 \mathrm{ab}$ & $106.20 \mathrm{~cd}$ & $10.81 \mathrm{~b}-\mathrm{d}$ & $22.81 \mathrm{~b}-\mathrm{d}$ & $3.75 e$ & $7.07 \mathrm{~b}$ \\
\hline $\mathrm{T}_{4}$ & $13.61 \mathrm{~b}$ & $5.00 \mathrm{ab}$ & $21.16 \mathrm{ab}$ & $107.50 \mathrm{~b}-\mathrm{d}$ & $10.05 \mathrm{~cd}$ & $24.10 \mathrm{ab}$ & $3.81 e$ & $7.09 \mathrm{~b}$ \\
\hline $\mathrm{T}_{5}$ & $12.42 \mathrm{~b}$ & $4.12 \mathrm{ab}$ & $20.67 \mathrm{~b}$ & $107.60 \mathrm{~b}-\mathrm{d}$ & $11.05 \mathrm{~b}-\mathrm{d}$ & $24.24 \mathrm{ab}$ & $3.78 e$ & $7.01 \mathrm{~b}$ \\
\hline $\mathrm{T}_{6}$ & $13.80 \mathrm{~b}$ & $4.40 \mathrm{ab}$ & $21.69 \mathrm{ab}$ & $108.00 \mathrm{bc}$ & $11.67 \mathrm{~b}$ & $22.03 \mathrm{~d}$ & $4.51 \mathrm{~d}$ & $7.07 \mathrm{~b}$ \\
\hline $\mathrm{T}_{7}$ & $12.33 \mathrm{~b}$ & $4.42 \mathrm{ab}$ & $23.45 \mathrm{a}$ & $107.60 \mathrm{~b}-\mathrm{d}$ & $10.74 b-d$ & $22.76 b-d$ & $4.60 \mathrm{~d}$ & $7.05 \mathrm{~b}$ \\
\hline $\mathrm{T}_{8}$ & $14.85 \mathrm{~b}$ & $4.53 \mathrm{ab}$ & $20.59 b$ & $108.80 \mathrm{~b}$ & $10.96 \mathrm{~b}-\mathrm{d}$ & $23.70 \mathrm{a}-\mathrm{c}$ & $4.70 \mathrm{~d}$ & $6.67 \mathrm{~b}$ \\
\hline $\mathrm{T}_{9}$ & $13.27 \mathrm{~b}$ & $4.67 \mathrm{ab}$ & $20.13 b$ & $108.40 \mathrm{bc}$ & $11.38 \mathrm{~b}$ & $22.92 \mathrm{~b}-\mathrm{d}$ & $4.87 \mathrm{~cd}$ & $6.79 \mathrm{~b}$ \\
\hline $\mathrm{T}_{10}$ & $13.65 \mathrm{~b}$ & $5.53 a$ & $20.61 \mathrm{~b}$ & $109.30 \mathrm{~b}$ & $11.18 \mathrm{bc}$ & $22.83 \mathrm{~b}-\mathrm{d}$ & $6.02 \mathrm{~b}$ & $6.83 \mathrm{~b}$ \\
\hline $\mathrm{T}_{11}$ & $15.03 \mathrm{~b}$ & $5.30 \mathrm{a}$ & $22.01 \mathrm{ab}$ & $108.10 \mathrm{bc}$ & $10.20 \mathrm{~cd}$ & $24.78 \mathrm{a}$ & $5.39 \mathrm{c}$ & $7.05 \mathrm{~b}$ \\
\hline $\mathrm{T}_{12}$ & $18.35 \mathrm{a}$ & $3.147 \mathrm{~b}$ & $21.90 \mathrm{ab}$ & $111.70 \mathrm{a}$ & $9.897 \mathrm{~d}$ & 23.57 a-d & $6.61 \mathrm{a}$ & $7.90 \mathrm{a}$ \\
\hline CV (\%) & 13.17 & 21.93 & 6.52 & 1.08 & 5.48 & 0.68 & 6.93 & 0.17 \\
\hline
\end{tabular}

$\mathrm{T}_{1}=$ Control (no NPK fertilizer), $\mathrm{T}_{2}=$ No nitrogen fertilizer, $\mathrm{T}_{3}=$ No phosphorus fertilizer, $\mathrm{T}_{4}=$ No potassium fertilizer, $\mathrm{T}_{5}=50 \%$ recommended dose of granular urea and 50\% PRH (fruit vinegar), $\mathrm{T}_{6}=50 \%$ recommended dose of phosphorus fertilizer and $50 \% \mathrm{PRH}$ (fruit vinegar), $\mathrm{T}_{7}=50 \%$ recommended dose of potassium fertilizer and $50 \% \mathrm{PRH}$ (fruit vinegar), $\mathrm{T}_{8}=50 \%$ recommended dose of NPK fertilizer and $50 \% \mathrm{PRH}$ (fruit vinegar), $\mathrm{T}_{9}=100 \%$ recommended dose of NPK fertilizer, $\mathrm{T}_{10}=100 \% \mathrm{PRH}$ (fruit vinegar), $\mathrm{T}_{11}=2.7 \mathrm{~g}$ urea super granules per 4 hills, $\mathrm{T}_{12}=2.7 \mathrm{~g}$ urea super granules per 4 hills and 50\% PRH (fruit vinegar).

From the above results it can be concluded that application of $2.7 \mathrm{~g}$ urea super granules per 4 hills and 50\% PRH (fruit vinegar) contributed to higher grain yield in Boro rice. 
Effect of NPK and plant Extracted Pyroligneous Acid on Yield of Boro Rice

\section{References}

BRRI (Bangladesh Rice Research Institute). 2000. Annual Report for 1999-2000. Bangladesh Rice Research Institute, Joydebpur, Gazipur. p.138.

Gomez, K. A. and A. A. Gomez. 1984. Duncan's Multiple Range Test. Statistical Procedures for Agricultural Research. 2nd Edn., A Wiley Inter-Science Publication, John Wiley and Sons, New York. pp.202-215.

IRRI (International Rice Research Institute). 2012. World Rice Statistics. [http://ricestat. irri.org:8080/wrs2/entrypoint.htm]

Kabir, M. H., M. A. R. Sarkar and A. K. M. S. H. Chowdhury. 2009. Effect of urea super granules, prilled urea and poultry manure on the yield of transplant aman rice varieties. J. Bangladesh Agril. Univ. 7(2): 259-263.

Masum, S. M., M. H. Ali and M. J. Ullah. 2008. Growth and yield of two T. aman rice varieties as affected by seedling number hill ${ }^{-1}$ and urea supergranules. J. agric. educ. technol. $11(1$ \& 2): 51-58.

Masum, S. M., M. Malek, M. S. H. Mandal, M. H. Haque and Z. Akther. 2013. Influence of plant extracted pyroligneous acid on transplanted aman rice. J. Expt. Biosci. 4(2): 31-34.

Mohanty, S. K., S. P. Chakravorti and A. Bhadrachalam. 1989. Nitrogen balances studies in rice using 15 N-labeled urea super granules. J. Agric. Sci. 113(1): 119-121. 